\title{
Several Influence Factors on Acute Hemorrhagic Conjunctivitis Diffusion within Airport Terminal with Agent-based SEIR Model
}

\author{
YIN Li ${ }^{1, a}$, LI Jing ${ }^{1, b}$ \\ ${ }^{1}$ Xinjiang Uygur Autonomous Regional Hospital of Traditional Chinese Medicine, Uygur, 830000, \\ China \\ aemail: 449744382@qq.com, bemail:824420lijing@163.com
}

\begin{abstract}
Keywords: Acute Hemorrhagic Conjunctivitis, Super Spreader, Social Relation Structure, Personal Contact Network, Agent-based SEIR Model
\end{abstract}

\begin{abstract}
Since the outbreak of pandemics, acute hemorrhagic conjunctivitis (AHC) has caused extensive attention in the field of public health. The purpose of this study was to quantitatively evaluate the influences of super spreader, passenger source and social relation structure on the AHC transmission in terminal. A method combining hierarchical structure of personal contact network with agent-based SEIR model was proposed to analyze the characteristics of AHC diffusion within terminal. Based on the spatial distance between individuals, the hierarchical structure of personal contact network was defined to construct a complex relationship of passengers in the real world. The agent-based SEIR model was improved by considering the individual level of AHC spread characteristics and influence of super spreader. Moreover, the characteristic of passenger movement was described by the improved social force model. To evaluate the method, this process was fused in simulation based on the constructed personal contact network, and personal contact network was defined by following four layers: social relation structure, subareas, space distribution, and the whole terminal. Super spreader and partition of passenger sources were found to impact a lot in departure, while social relation structure imposed a great influence in arrival. It contributes to proposing effective AHC measures by airport relevant department and improving the efficiency and ability of epidemic prevention on the public health.
\end{abstract}

\section{Introduction}

In recent decades, acute hemorrhagic conjunctivitis (AHC) has grown to be a global contagious eye disease. It is first occurred in many nations and areas in 1967, such as Africa, Asian, Europe and America and so on, and popular every other three or four years [1]. Since the high-density of passengers in airport terminal, the possibility of personal interact is large increase, which provides a quite convenient approach for AHC transmission. Therefore, more and more scholars pay close attention to the spread characteristic and control measures of acute hemorrhagic conjunctivitis. In fact, the spread of AHC is interpersonal. To study the impact of individual contact behavior on AHC transmission, hierarchical structure of personal contact network is more suitable for the evaluation of spatial information in small space. Therefore, we require a hierarchical spatial-topology structure to model personal contact network. It is feasible for analysis of AHC transmission in terminal.

On foundations above, we propose a method to quantitatively analyze the influences on AHC transmission in terminal by integrating agent-based SEIR model with hierarchical structure of personal contact network. The agent-based susceptible-exposed-infectious-removed (SEIR) model is used to describe dynamics of AHC transmission mechanism, and passenger social relation structure is captured and quantified by utilization of hierarchical network of personal contact. The characteristic of passenger movement is described by the improved social force model so as to quantitatively analyze the phenomenon of super spreaders. We show that, with this method, it is possible to study the impact of super spreader, passenger sources and social relation structures on AHC diffusion. To evaluate the proposed method, a hierarchical personal contact network of a terminal is built based on passenger geospatial distribution. Transmission experiments of AHC are 
carried out in the generated network to simulate AHC evolution process. Besides, we analyze the impact of passenger topological character and spatial clustering of AHC spread. In consequence, it contributes to proposing effective AHC measures by airport relevant department and improving the efficiency and ability of epidemic prevention on the public health.

\section{Methods}

\section{Probability distribution function of AHC transmission}

Acute hemorrhagic conjunctivitis is an infectious disease caused by intestinal virus, which is more common in summer and autumn. Individuals at each age group have the possibility to be infected. Moreover, contacts among individuals are considered as the major route of AHC spread, such as touching the hands, water or items contaminated by someone infected. Therefore, AHC possess the characteristics of short latency, strong infectiousness and quickly spread, and which lead to increase the likelihood of its outbreak.

The spread possibility correlates valid contact time [2]. $\beta$ is a constant, represent the infect rate when individual contact with pathogen. The spreading probability density function- $f(t)$ changes along with valid contact time $t$, has to follow Eq. (1) and Eq. (2). Sequentially, Eq. (3) is derived. Then probability distribution function $F(t)$ is given in Eq. (4).

$$
\begin{aligned}
& f(t)=\beta\left(1-\int_{0}^{t} f(\tau) d \tau\right)(0 \leq \beta \leq 1) \\
& \int_{0}^{+\infty} f(t) d t=1 \\
& f(t)=\beta \exp (-\beta t)(0 \leq \beta \leq 1) \\
& F(t)=1-\exp (-\beta t)(0 \leq \beta \leq 1)
\end{aligned}
$$

\section{Influential factors needs to be considered}

\section{Super spreader phenomenon}

In terminal, passengers flow on specific directions and are more incline to the contacts induced from their own departure or arrival path. And there are much more procedures or services needed to be carried out in terminal. Conducting these procedures lessens the passenger interval and raises the likelihoods of getting contaminated. Furthermore, staffs in the terminals frequently contact with passengers. Once a staff in a terminal gets sick, it is highly possible for him becoming a super spreader which is the so-called super spreader phenomenon. AHC would get an extensive spread due to super spreader. Consequently, the control of contact behaviors with super spreader plays a key role in AHC diffusion intervention within terminal.

\section{Passenger sources partition}

Population mobility is one of the important factors which push the fast AHC transmission. The floating population flows among different cities using vehicle as their carriers. Furthermore, air travel has greatly accelerated AHC diffusion and other diseases transmitted by person-to-person contact [3]. At the same time, terminal has a very mobile population, and gathers passengers from different regions. For this reason, we divide passenger sources into affected areas and unaffected areas. Passengers coming from affected area carry AHC virus into local. Moreover, the airport services and procedures make passenger interval reducing, while the possibility of getting infected increasing. Therefore, AHC virus would be extensively spread due to the daily population flows coming from affected areas. Based on these, the control of interactive behaviors among passengers from affected area and unaffected areas plays a critical role in AHC spread intervention.

\section{Social relation structure}

Social relation structure represents the frequent degrees of contact relation among passengers. It is impossible for one passenger to contact all passengers at the same time. Therefore, in order to identify the effective contact for AHC virus transmission, we classify social relation structures [4] of passengers into some categories based on the spatial distance between individuals [5], namely, relatives, colleagues, friends and other types of interpersonal relationship, which results in the spread of AHC in social network. Moreover, passengers in different social relation structures have 
diverse intimate relationships. Walking characteristics among passengers with different relationships are distinctly various, which lead to the difference in valid contact time $t$ in Eq. (4). In the research of AHC spread, complex interpersonal relationship is extracted from hierarchical structure of personal contact network.

\section{Model modifications}

\section{Agent-based SEIR model}

Agent is an individual with sociality, autonomy and activity. With the change of environment, they alter their behaviors and states according to changes in their environment [6]. Rules of agent-based SEIR model for AHC in terminal are improved as follow:

First, there were four mutually exclusive disease states during the diffusion, $\mathrm{S}$ (susceptible), $\mathrm{E}$ (exposed), I (infectious) and R (removed) [7]. The transition process of four disease states is shown as Figure 1. Second, as to susceptible, who can be contaminated into exposed state, Eq. (4) determined the relationship between infected probability $F(t)$ and valid contact time $t$ [8-9].Third, the exposed could only remain latent during the residence or leave. Fourth, infectious passengers might infect susceptible pedestrian into exposed during their stay in terminal. Finally, removed were not capable of infecting or being infected.

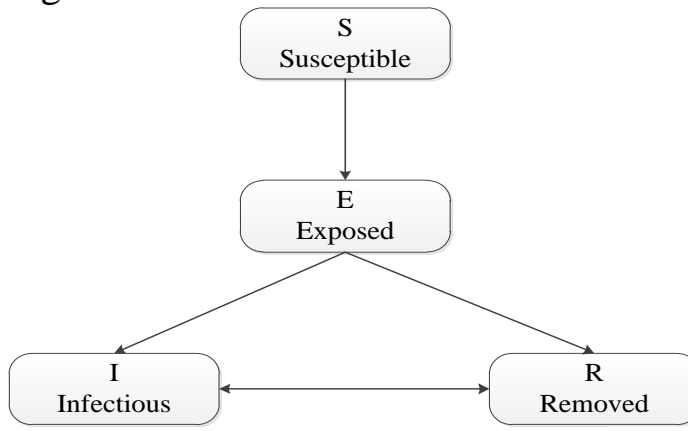

Figure 1 The transition process of four disease states

\section{Hierarchical structure of personal contact network model}

The hierarchical structure of personal contact network is composed of multi-layer networks, and each layer contains several independent networks with same attributes are contained in each layer [5].

The first layer of hierarchical network $(\alpha=0)$ is the unit model, which describes the most frequent contact relations. Individuals in a unit model have a high likelihood to contact each other. We use node number $\mathrm{n}$ to control network topology. All the unit models in a whole network is a set $\left\{n_{i}\right\}$. Each unit has a high clustering coefficient $C=1$. The number of basic unit model $\mathrm{M}$ is:

$$
M=\frac{N}{n_{0}}
$$

where $N$ is number of nodes in a personal contact network. $n_{0}$ is the number of nodes in all the unit models.

Based on the unit model, we can construct the hierarchical network of personal contact. It connects nodes from unit model to the whole hierarchal contact network. The newly constructed network $W$ is as follow:

$$
\left\{\begin{array}{l}
U=u_{1} \cup u_{2} \cup \cdots \cup u_{i} \\
V=v_{1} \cup v_{2} \cup \cdots \cup v_{i}+v_{\text {new }}, \quad i \in\left[1, n_{x}\right] \\
W=(U, V)
\end{array}\right.
$$

Where $u_{i}$ and $v_{i}$ denote node set and edge set in a unit model $i$ respectively. $v_{\text {new }}$ represents the newly added edges. $n_{x}$ is the number of unit models that compose the higher layer network. $U$ and $V$ mean all nodes and edges in network $W$.

Here, to fix the number of sub-networks, we use both spatial distance and social relation structure to estimate number of abstracted layers and number of sub-networks in a layer. Besides, we define a probability distribution function $F(y)$ [5] to decide whether to add a new edge between individual $i$ and individual $j$ in different sub-networks. 
$F(y)=\frac{e^{-\alpha}\left(\frac{d_{i}}{d_{i \max }}+\frac{d_{j}}{d_{j \max }}\right)}{2}$

In which, $d_{i}$ is the actual degree of individual $i$ in layer $\alpha . d_{i}$ max expresses the maximum degree of links that individual $i$ owns in layer $\alpha-1$.

The spread of AHC takes on the characteristics of hierarchical structure of personal contact network model. In the investigated terminal, personal contact networks are defined by following four layers: social relation structure $(\alpha=0)$, subareas $(\alpha=1)$, space distribution $(\alpha=2)$, and the whole terminal $(\alpha=3)$, which is shown as Figure 2. Details will be shown in the experiments later.

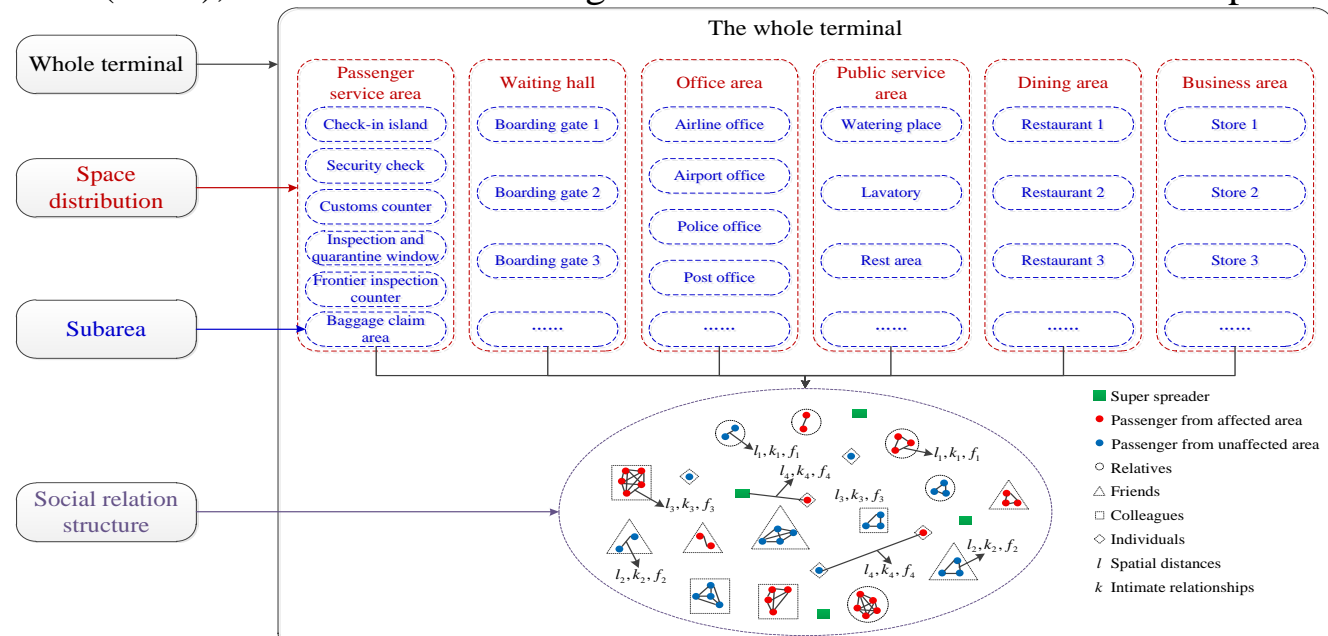

Figure 2 The hierarchical structure of personal contact networks in terminal

\section{Improved social force model}

The basic characteristics of social force model are as follows [10]:

Suppose a person $i$ with weight $m_{i}$ expects to walk at speed $v_{i}^{0}$ and direction $\vec{e}_{i}^{0}$. When accelerating to $\vec{v}_{i}$ in $\tau_{i}$ time, social force model argue that a certain distance with other pedestrians and walls needs to be maintained, which relates to velocity and could be modeled by psychological forces $\left(\vec{f}_{i j}\right.$ and $\vec{f}_{i w}$ ).

Generally, pedestrian $i$ tends to remain a certain distance with $j$. This psychology could be expressed by $\vec{f}_{i j}$ :

$$
\left\{\begin{array}{l}
\vec{f}_{i j}=f_{i j n} \vec{n}_{i j}+f_{i j t} \vec{t}_{i j} \\
f_{i j n}=A_{i} \exp \left[\left(r_{i j}-d_{i j}\right) / B_{i}\right]+k g\left(r_{i j}-d_{i j}\right) \\
f_{i j t}=\kappa g\left(r_{i j}-d_{i j}\right) \Delta v_{j i}^{t}
\end{array}\right.
$$

Where, the normal and tangential component of $\vec{f}_{i j}$ are comprised of $f_{i j n}$ and $f_{i j t}$. $\vec{n}_{i j}=\left(n_{i j}^{1}, n_{i j}^{2}\right)=\left(\vec{r}_{i}-\vec{r}_{j}\right) / d_{i j}$ and $\vec{t}_{i j}=\left(-n_{i j}^{2}, n_{i j}^{1}\right)$ stand for the normal and tangential standardized vector pointing to $j$ from $i . A_{i}, B_{i}, k$ and $\kappa$ are constants, while $A_{i}$ denotes interactional strength among pedestrians, $B_{i}$ represents psychological repulsion scope of pedestrians. The sum of radius of pedestrian $\mathrm{i}$ and $\mathrm{j}$ is expressed by $r_{i j}=r_{i}+r_{j}$ while $d_{i j}$ is the centroid distance between pedestrian $i$ and $j \cdot \Delta v_{j i}^{t}=\left(\vec{v}_{j}-\vec{v}_{i}\right) \Delta \vec{t}_{i j}, \quad g(x)=\left\{\begin{array}{l}0, x \leq 0 \text { set the negative value to } 0 . \\ x, x>0\end{array}\right.$

Similarly, the force $\vec{f}_{i w}$ between pedestrians and obstructions is given by:

$$
\left\{\begin{array}{l}
\vec{f}_{\text {iw }}=f_{\text {iwn }} \vec{n}_{\text {iw }}+f_{\text {ivt }} \vec{t}_{\text {iw }} \\
f_{\text {iwn }}=A_{i} \exp \left[\left(r_{i}-d_{i w}\right) / B_{i}\right]+k g\left(r_{i}-d_{i w}\right) \\
f_{\text {ivt }}=\kappa g\left(r_{i}-d_{i w}\right)\left(\vec{v}_{i} \Delta \vec{t}_{i w}\right)
\end{array}\right.
$$


Where, the normal and tangential component of $\vec{f}_{i w}$ are comprised of $f_{\text {iwn }}$ and $f_{\text {iwt }}$. The normal and tangential unit vector of obstruction w pointing to pedestrian $i$ are represented by $\vec{n}_{i w}=\left(n_{i w}^{1}, n_{i w}^{2}\right)$ and $\vec{t}_{i w}=\left(-n_{i w}^{2}, n_{i w}^{1}\right) \cdot d_{i w}$ stands for the distance from centroid of pedestrian $i$ to the surface of obstruction $w$. The rest parameters remain the same as Eq. (8).

Besides, the small groups with a certain connection will walk together. We think the close connections among small group needed to get special considerations. The force $\vec{f}_{i q}$ is employed to express these relations:

$$
\left\{\begin{array}{l}
\vec{f}_{i q}=f_{i q n} \vec{n}_{i q} \\
f_{i q n}=\omega \exp \left[\left(r_{i q}-d_{i q}\right) / l\right]
\end{array}\right.
$$

Where, $\vec{n}_{i q}=\left(n_{i q}^{1}, n_{i q}^{2}\right)$ denotes the unit vector pointing to pedestrian $i$ from $q$. The distance from pedestrian $i$ to $q$ is represented by $d_{i q}$ while $\omega$ and $l$ are constants. $\omega$, a positive number, stands for attraction intensity among accompanies, that the intimate relationships coefficient. $l$ represents attraction scope, that the spatial distances. The other parameters remain the same as Eq. (8).

Hence, the acceleration in moment t could be improved by kinematical equation given by Eq. (11) [11], where the first term denotes the self-driving force. The pedestrian accelerates or decelerates due to the joint forces of these four forces.

$$
m_{i} \frac{d \vec{v}_{i}}{d t}=m_{i} \frac{v_{i}^{0}(t) \vec{e}_{i}^{0}(t)-\vec{v}_{i}(t)}{\tau_{i}}+\sum_{j(\neq i)} \vec{f}_{i j}+\sum_{w} \vec{f}_{i w}+\sum_{q} \vec{f}_{i q}
$$

The interactions among individuals of the four kinds of passengers are shown by Figure 2.

\section{Results}

\section{Experimental groups and parameter initialization}

To validate the model mentioned above, we carry out five sets of simulative experiments to study the impact of AHC diffusion factors. They are (1) considering super spreader phenomenon only, (2) considering passenger source partition only, (3) considering social relation structure only, (4) without considering the three factors mentioned above, and (5) taking account of super spreader phenomenon, passenger source partition and social relation structure at the same time.

In the five experiments, we set the initial percentages of passengers' states based on AHC spread characteristic in airport terminal, which respectively are 78.6\% (susceptible), 8.5\% (exposed), 3.3\% (infectious) and 9.6\% (removed) both in arrival and departure. In each experiment, the total number of passengers in arrival and departure are roughly 8240, among which the number of domestic passengers is more than that of international passengers, approximately 3.8 times.

\section{Assumptions of influential factors}

\section{Super spreader phenomenon assumptions}

Super spreaders increase the likelihood of passengers being infected. So, the number of super spreaders in each procedure plays an important role in AHC diffusion. Table 1 lists the number of assigned super spreaders in the experiments.

Table 1 Number of assigned super spreaders

\begin{tabular}{ll}
\hline Scene & Super spreader \\
\hline \multirow{2}{*}{$\begin{array}{l}\text { Departure } \\
\text { Arrival }\end{array}$} & $\begin{array}{l}\text { Domestic check-in } \times 3 \text {, Domestic security check } \times 2 \text {, Domestic boarding gate } \times 2 \text {, International security } \\
\text { Store } \times 4 \text {, Restaurant } \times 3\end{array}$ \\
Customs $\times 3$, Frontier inspection $\times 2$
\end{tabular}

\section{Passenger sources partition assumptions}

The initial number of affected area passengers has made a great impact on the evolution process of AHC transmission. Table 2 lists the number of passengers coming from affected area in departure and arrival. 
Table 2 The number of passengers coming from affected areas

\begin{tabular}{lll}
\hline Scene & Passenger source partition & Passenger number \\
\hline Departure & Affected areas & 20 \\
Arrival & Affected areas & 20 \\
\hline
\end{tabular}

\section{Social relation structures assumptions}

History statistics shows that the proportions of passengers classified by social relation structure above are 36.2\% (relatives), 22.4\% (friends) 18.9\% (colleagues), and 22.5\% (individuals) respectively [12]. Table 3 provides the assumptions about the four social relation structures of passengers.

Table 3 The assumptions of social relation structure

\begin{tabular}{llllll}
\hline $\begin{array}{l}\text { Classification of } \\
\text { passengers }\end{array}$ & Passenger number Spatial distances & $\begin{array}{l}\text { Intimate } \\
\text { relationships } \\
\text { coefficient } \omega\end{array}$ & $\begin{array}{l}\text { Interactional } \\
\text { strength } A_{i}\end{array}$ & $\begin{array}{l}\text { Psychological } \\
\text { repulsion scope } \\
B_{i} / \mathbf{m}\end{array}$ \\
\hline Relatives & uniform $(2,3)$ & $0 \sim 0.55$ & 2.6 & 0.3 & $1.2 \sim 3.6$ \\
Friends & uniform $(2,5)$ & $0.55 \sim 0.8$ & 1.8 & 0.3 & $1.2 \sim 3.6$ \\
Colleagues & uniform $(2,8)$ & $0.8 \sim 1.2$ & 1.5 & 0.3 & $1.2 \sim 3.6$ \\
Individuals & 1 & $1.2 \sim 3.6$ & 0 & 0.3 & $1.2 \sim 3.6$ \\
\hline
\end{tabular}

\section{Hierarchical structure of personal contact network model in terminal}

We have made a survey on the social relation pattern of passengers in a Chinese terminal to get their spatial distribution. The investigation data included: the number of passengers in each social relation structure, the number of subareas in each region, the number of space distribution in each terminal. Then, we utilize these data to initialize the hierarchy of personal contact networks. The four layers of personal contact network are shown as Figure 3.

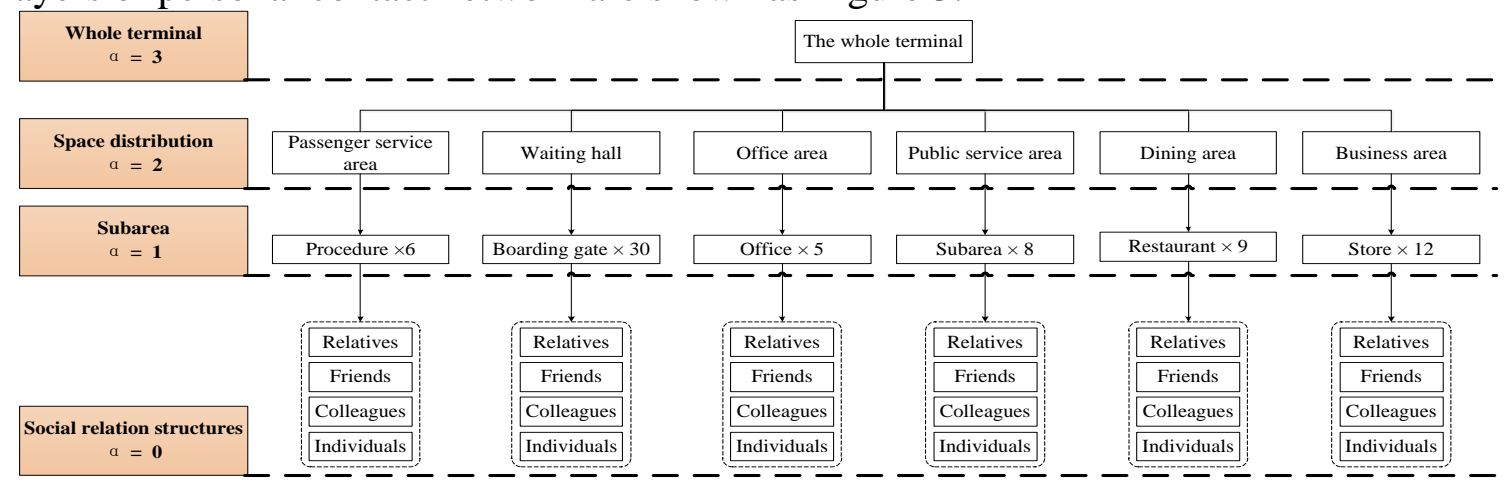

Figure 3 The four layers of personal contact network setting

\section{Terminal plane structure and passengers' flow setting}

The plane layout of a terminal is given as follow. Among them, Figure 4 (left) shows the plane layout of departure, where the entrance -- 4 , check-in -8 , security check - 55 , customs inspection -6 , inspection and quarantine window -- 6, immigration inspection -- 13 , and boarding gate - 63 , are offered. Figure 4 (right) is the plane layout of arrival, in which the inspection and quarantine -- 16, immigration inspection -- 20, customs inspection -- 3, baggage claim -- 15, and exit -4 , are represented.

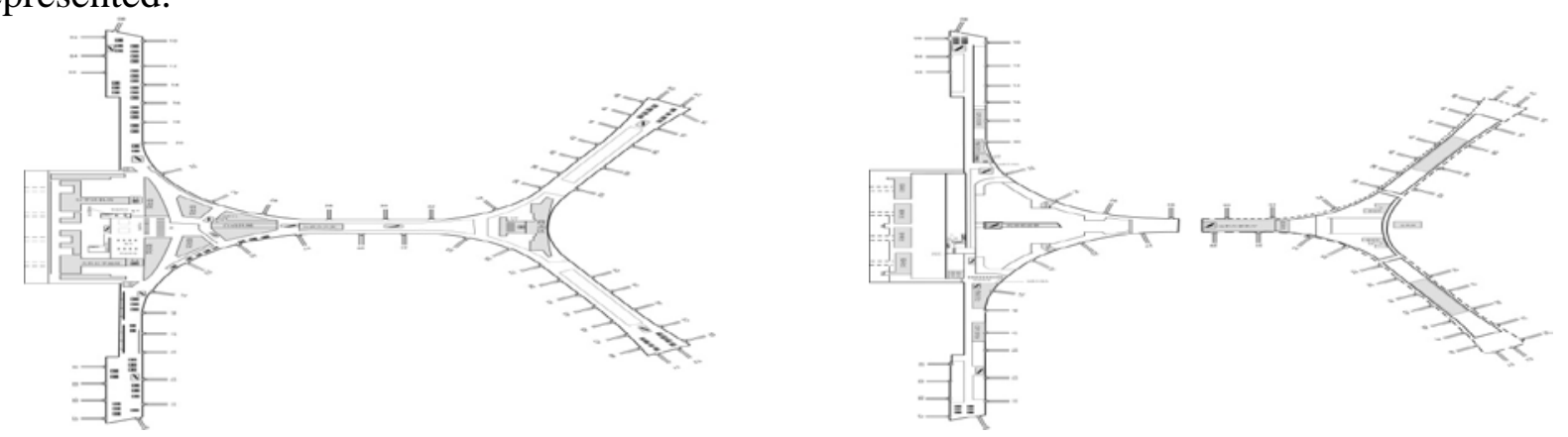

Figure 4 The plane layout of the terminal 


\section{Discussion}

To identify the influence of super spreader, passenger source and social relation structure on AHC transmission in terminal, we carry out AHC transmission experiments by utilization of proposed model with consideration of super spreader, passenger source and social relation structure, respectively.

The average numbers of infectious passengers both in departure and arrival vary with time. They are respectively shown as Figure 5 (left) and Figure 5 (right). In the departure experiments, there are average 26.4 people infected by each super spreader, while 1.56 people contaminated by an ordinary infectious. Whereas, there are average 13.7 people contaminated by each super spreader and 0.83 people get contaminated by common infectious people in arrival whose data is given by Figure 5 (right). Furthermore, the total number of infectious passengers increases by 334 in departure all day while it only grows 34 in arrival considering super spreader. Consequently, we argue that super spreader impacts a great on prevalence in departure.

In the departure experiments, there are average 25.9 people infected when considering passengers coming from affected area, while 1.26 people contaminated by infectors without considering passenger source. Whereas, in the view of arrival experiment, 5.18 people get contaminated when passengers come from affected area and there are 0.34 people infected by infectious people ignoring the passenger sources. Furthermore, the total number of infectious passengers increases by 299 in departure all the day while it only grows by 42 in arrival in the consideration of passenger source partition. Hence, we argue that source of AHC virus carrier impacts a great on AHC spread in departure.
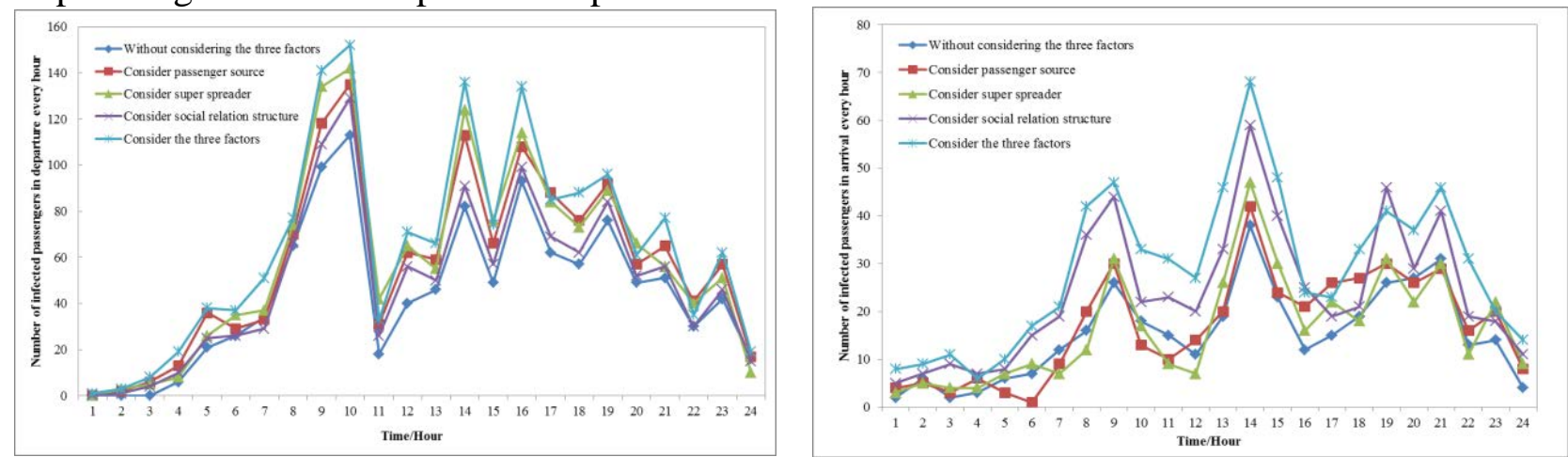

Figure 5 The number of infected passengers in departure (left) and arrival (right) every hour

The total number of infected passengers in departure and arrival are provided in Table 4. Contrast with the experiment irrespective of the three factors, the number of infectious passengers in departure experiment considering social relation structure grows by 117, while increases by 211 in arrival. And the experiment reveals that social relation structure plays an important role in arrival. Due to the different procedures in departure and arrival, passengers will get together to check-in or security check no matter what social relation structures they are in departure. Therefore, infectious passengers have more chances to contact and infect others. However, passengers of different social relation structures will separate after they disembark in arrival, which causes the reduction of the possibility of being infected.

Table 4 The total number of infected passengers in departure and arrival

\begin{tabular}{lllll}
\hline & $\begin{array}{l}\text { Without considering Considering } \\
\text { the three factors } \\
\text { passenger source } \\
\text { partition }\end{array}$ & $\begin{array}{l}\text { Considering super } \\
\text { spreader } \\
\text { phenomenon }\end{array}$ & $\begin{array}{l}\text { Considering social } \\
\text { relation structure }\end{array}$ & $\begin{array}{l}\text { Considering all the } \\
\text { three factors }\end{array}$ \\
\hline Departure 1075 & 1374 & 1409 & 1192 & 1564 \\
Arrival 365 & 407 & 399 & 576 & 693 \\
\hline
\end{tabular}




\section{Conclusions}

In this paper, we propose a novel approach to organically combine hierarchical structure of personal contact network model with agent-based SEIR model based on individuals' spatial distribution information in terminal. By analyzing and simulating the different factors that affect AHC virus spread, we reveal the characteristics and evolution process of AHC transmission.

(1) The proposed method possessed the capability of quantitatively describing the AHC prevalence process, analyzing influences of concerned factors, as well as proposing effective intervention strategies.

(2) The dynamic evolution of AHC transmission is simulated based on the experiments. In the spread process, super spreader and passenger sources are found having marked impact in departure, while social relation structure imposes a great influence in arrival.

(3) Endemic area isolation and should be implemented in infectious groups. Other intervention measures, such as terminal ventilation, disinfection and reasonable partition and opening more service resources as earlier as possible, should be adapted to the arrest AHC development in both departure and arrival.

\section{Acknowledgement}

In this paper, the research was sponsored by the National Natural Science Foundation of China (Project No. 71303110)

\section{References}

[1] Zhao LQ, Luan YM, Liu SJ. Epidemiological investigation of acute hemorrhagic conjunctivitis in Haizhu district of Guangdong province in 2007[J]. Occup and Health, 2008, 24(23): 2556-2557.

[2] Zhang H, Chen C, Wang CC. Epidemic spread and control on complex networks using cavity theory[J]. Journal of University of Electronic Science and Technology of China, 2011, 40(4): 491-496.

[3] Kenah E, Chao DL, Matrajt L, et al. The global transmission and control of influenza[J]. PLoS One. 2011;6(5):e19515.

[4] Liang ZS. Research on Simulation Platform of Influenza A (H1N1) Based on Multi-Agent Complex Network AND GIS[D]. Kunming, China: Yunnan Normal University; 2011.

[5] Ge YZ, Song ZC, Qiu XG, Song HB, Wang Y. Modular and hierarchical structure of social contact networks. Phys A Stat Mech Appl. 2013; 392 (19):4619-28.

[6] Bu FL, Feng PY. Analysis of agent-based "non-organization and non-direct interest" collective events[J]. Emergency Management and Management Sciences (ICEMMS), 2011 2nd IEEE International Conference on. IEEE, 2011; 417-421.

[7] Watts, Duncan J., et al. Multiscale, resurgent epidemics in a hierarchical metapopulation model[J]. Proceedings of the National Academy of Sciences of the United States of America ; 2005;102(32): 11157-62.

[8] Simoes, J. M. An Agent-Based Approach to Spatial Epidemics through GIS[D]. University College London, 2006.

[9] James, A., Pitchford, J.M. et al. An Event-based Model of Super spreading in Epidemics[J]. Proceedings of the Royal Society Biological Sciences, 2007, 274(1610): 741-7.

[10] Helbing, Dirk, Illes Farkas, and Tamas Vicsek. Simulating dynamical features of escape panic[J]. Nature ,2000;407(6803): 487-90. 
[11] WANG, Lei, Yun CAI, and Qing XU. Modifications to Social Force Model[J]. Journal of Nanjing University of Science and Technology (Natural Science) ,2011:35(1): 144-9.

[12] Civil Aviation Domestic Passenger Market Characteristics Research Report for 2010[J]. Beijing: Civil Aviation Management Institute of China, Institute of civil aviation transportation market, 2010; pp 55-63. 